\title{
Requirements and Metrics for Location and Tracking for Ambient Assisted Living
}

\author{
Samih Eisa, Adriano Moreira \\ Mobile and Ubiquitous Systems research group, Centro Algoritmi, \\ University of Minho, Guimaraes, Portugal \\ sameheisa@gmail.com, adriano.moreira@algoritmi.uminho.pt
}

\begin{abstract}
Location and tracking services and technologies are becoming fundamental components for supporting healthcare solutions. They facilitate patients' tracking and monitoring processes and also allow for better and long-term daily activity recognition. Various location and tracking services have been developed, over the last years, to provide real time localization for different applications. However, most of these services are not designed particularly to comply with all the requirements of Ambient Assisted Living (AAL) and, as a result, they reduce the viability of adopting AAL services as an alternative for continuous healthcare services. In this paper we set out the general requirements for location and tracking services for AAL. The requirements are extracted from a typical scenario of AAL. From the scenario, we define the requirements and also we identify a set of metrics to be used as evaluation criteria. If the identified requirements and metrics are adopted widely, potential location and tracking services will fit the real needs of AAL, and thus will increase the accessibility to AAL services by a larger sector of people. Moreover, in the paper, we evaluate two of the existing location techniques through the use of the proposed metrics. The aim is to asses to which level these solutions fulfill the identified requirements.
\end{abstract}

Keywords- AAL requirements; home environments; indoor Location and tracking;

\section{INTRODUCTION}

The demographic change we are witnessing in all over the world, especially in Europe, indicate that most of the industrial and developed countries are facing a great challenge of having more aging population. Projections indicate $30 \%$ of the population in Europe alone will be over 65 in 2060 [1], while in Japan, the percentage is even higher: it will increase to $40 \%$ in 2055 [2]. This continued growth, indeed will have a great economical impact and will increase the need for more care services for senior citizens, especially for those who are living alone and having chronic illnesses or impairment diseases. Managing chronic illnesses of the elderly people requires long-term hospitalization and personal care which result in adding massive costs to the healthcare sector. Thus, an efficient and cost-effective solution is becoming a must for most of the countries. Governments and caregivers alike are trying to find replacement of the conventional way of care with a more efficient and effective solution that allows elderly people to maintain a certain degree of autonomy while their health status being continuously observed to avoid abnormalities in their daily life routines. Abnormalities include critical situations such as heart attacks, strokes, and sudden falls. In fact, such medical situations caused more deaths among senior citizens in the last few years and according to the World Health Organization (WHO), 30\% of the seniors fall every year and, as a consequence, $20-30 \%$ of them suffer serious injuries that reduce their mobility and increase the risk of death $[1,3]$. Within this context, there is a truly need for an intelligent environment to support the protection of elderly people. The environment should take into account their autonomy and independence requirements and also should be able to keep track of their daily life routines to avoid and alert for emergency situations. One possible solution to cope with such challenge is the use of Ambient Assisted Living (AAL). AAL refers to the use of ICT (Information and Communication Technology) for providing technology-based assisted living facilities for people with disabilities or chronic illnesses [4]. The facilities are provided in a proactive and preventive way of care, with remote coordination ability, to ensure health, safety, and well-being of the assisted persons and also reduce the cost of personal care services.

To support AAL, different location and tracking technologies are being exploited in many AAL systems. Technologies like WiFi, Global Positioning Systems (GPS), Ultra Wide Band (UWB), ultrasound, Camera-based, and Infrared Radiation (IR) have been widely used for tracking people inside/outside houses[5-7]. Based on these technologies, various commercial and experimental systems have been developed such as Ekahau [8], Microsoft RADAR [9], Active bat [10], Active badge [11], and Ubisense [12]. Most of these systems use some sort of wearable devices such as wearable tags, bracelets, pendants, or sensors like cameras, and motion sensors to detect peoples' locations. Many other technologies are also being integrated into advanced AAL systems, such as wearable sensors to monitor vital signs, or teleconferencing to combat loneliness, but these other technologies are outside the scope of this paper. Even though the existing location and tracking services are considered a great step-forward for providing location knowledge, they are not quite adequate for AAL. The design principles of most of these services are not fully matched with all the requirements of AAL. For instance, most of these services require a considerable number of location sensors/devices to be deployed in the environment in order to facilitate the collection of location data (e.g. RFID and ultrasound technologies). This, however, has led to a non-trivial increase in the installation effort and the overall cost for deploying the service. AAL services are mostly intended to be used in houses/residential environments by non-technical elderly 
people. Thus, the relevant option for such environments has to be cost-effective, efficient, and easy to install with more consideration to the technology acceptance of the assisted persons. Moreover, some of the existing location services make use of more advanced sensors (e.g. camera, microphone, and body-attached sensors) which also might not be desirable to most of the elderly people due to inconvenience and privacy issues. In fact, there are many requirements that have to be taken into account for location and tracking for AAL.

In this paper, we discuss the requirements for AAL location and tracking services. We cover a set of requirements extracted from a typical scenario of AAL system. We then present some of the possible assessment metrics that could be used to evaluate the existing localization techniques. Then, we apply those metrics to two of the existing techniques to assess to which level they fulfil the identified requirements. In this paper we are contributing to the definition of a generic evaluation framework for AAL location services. This framework is to be used not only for evaluation but, mostly, for providing guidelines for technological developments and system design.

The reminder of this paper is organized as follows. Section II presents the requirements for location and tracking for AAL. Section III discusses briefly the evaluation metrics and shows how these metrics could be used for assessment purposes. Section IV includes a comparison study of two of the existing location techniques. Section $\mathrm{V}$ concludes the paper and identifies some possible future research directions.

\section{REQUIREMENTS}

In this section we discuss the requirements for $\mathrm{AAL}$ location and tracking services. Noteworthy, this section does not include requirements for remote monitoring systems instead it focuses only on defining the most relevant requirements for locating people at home/residential environments. To help defining the requirements we provide the following scenario.

\section{A. Scenario}

We assume that there is a group of elderly people who are suffering from chronic illnesses or impairments diseases. Those elderly, as most elderly people, like to maintain their own life and live more autonomously in spite of their health status. The possible solution for many families and caregivers is to have an AAL system to monitor the daily life activities of those elderly while they are in their houses/apartments or even when they go outside. The system will act as a housemate for those elderly and will play an important role in avoiding abnormalities in their daily life routines and also help in increasing their safety feelings. The living environment of those elderly is characterized as a simple home environment in which all buildings are made of concrete and wooden structure with interior designs made according to the personal preferences of those elderly.

The AAL system will be required to provide seamless healthcare for those elderly with no significant interference in their daily activities like cooking, bathing, showering, and walking. For that, the system consists of an arbitrary number of location and tracking sensors distributed throughout the house and in the areas nearby. Sensor devices are embedded into the construction of the house and also they are connected to each other via wire/wireless networks. Sensors are arranged with respect to the house's structure and the personal preferences of the elderly. Due to privacy considerations, only simple sensors (e.g. motion sensors) should be used. Camera and microphone sensors should be avoided as most of those elderly will probably refuse to be monitored by these sensors. Location data collected from the sensor devices are aggregated in a local personal computer located in each house or alternatively, located in a place shared by a group of houses. Existing routers for Internet access or existing TV set-top boxes can be exploited as alternatives to a dedicated computer. The personal computer works as a gateway to transfer the aggregated raw data to a remote processing server located at a central medical unit where a group of medical specialists are working 24/7 daily to provide healthcare assistance and timely response in emergency situations. Moreover, a set of wearable tags, attached to those elderly, can also be used to help in tracking their locations whenever they go outside houses. Also, some relatives and neighbours are part of the monitoring process. They receive alarm notifications from the system in their mobile phones or portable devices to provide first aid assistance.

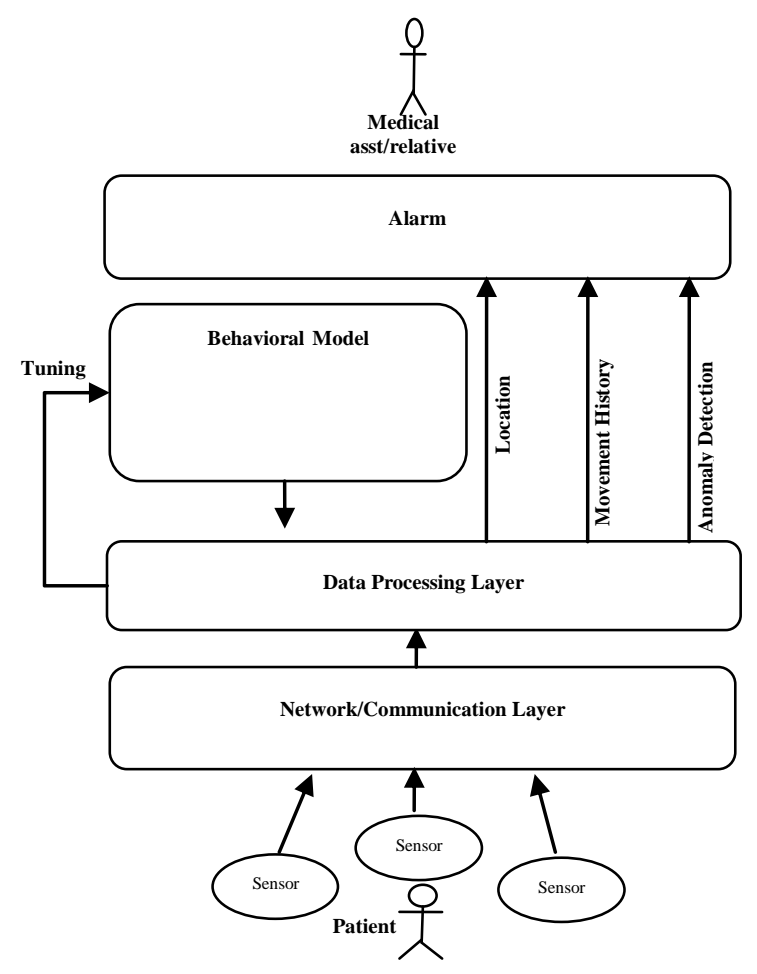

Figure 1. Conceptual view of a potential system for AAL

Figure1 illustrates the conceptual view of a potential system for AAL, adapted for the previously described scenario. In the figure, there are three core layers. The network and communication layer which is responsible for providing 
connectivity between the components of the system, the data processing layer, and the alarm layer which represents the application layer. Based on the system's architecture, these three layers collaborate to aggregate raw data from sensors, process these data, and extract contextual features to be delivered to the alarm layer as location information, movement history, and anomaly detection patterns. Moreover, a utility behavioural model is used for patterns extraction and a tuning process to continuously update this model.

Considering the previously described scenario, the following are some of the possible observed requirements for location and tracking services for AAL. The requirements are grouped into five categories: Functional, Non-Functional, Interface, Performance, and Product requirements.

\section{B. Functional Requirements}

This category includes all requirements that capture the intended behaviour of the service. This can be expressed as all tasks and functions the service is required to perform:

- Person Location: The service should be able to locate and track a person in an indoor environment.

- Person Identifier: Unique identifier for the tracked person is required to differentiate users in case multiple users are tracked simultaneously.

- Remote Transmission: The service should support the transmission of the location data to a remote server.

- Output Format: The service should be capable of converting the raw location data into human-interpreted format (e.g. Kitchen, bedroom, parking, etc.). Absolute or any other type of location output format will not be relevant.

- Presence/Absence detection: Whenever an absence of a user is detected or a user is out of the pre-defined perimeter, the service should have the ability to notify the remote operator indicating the event.

- No Movement detection: No movement for a time longer than a certain threshold value is considered an indicator of having a health problem (e.g. sudden falls), and thus the service should be able to indicate this event as well.

- Safety: The service should help to increase the safety feelings of the tracked persons. For instance, reducing the fear feeling of getting lost or wondering whenever a person goes outside the house for a walk or a visit. This can be achieved by providing on-demand calls for help (e.g. tags with on-demand call buttons).

\section{Non-Functional Requirements}

This category includes the non-functional requirements of the service. It specifies the criteria that can be used to judge the operation of the service.

- Coverage Scope: The required localization coverage of the service is inside and outside houses. All areas inside the house are required while only the areas that are nearby the house are required (e.g. locate a person at the garden or neighbors' houses).
- Resilience to power outage: Power outage occurrences might be quite common especially in houses or residential environments and therefore, the service should take this important requirement into account.

- Communication media: The communication link between the house's gateway and the remote server has to be fast and at reasonable cost. The communication requirements shall be as specified in ISO/IEEE 11073 family of standards. This standard provides a normative definition for controlling information exchange to and from personal healthcare devices and computer engines (e.g. cell phones and personal computers).

- Privacy: Privacy is extremely needed to ensure anonymity of the user. Simple sensors should be used; avoiding the use of camera sensors. This requirement aligns with the need for developing cheapest location service.

\section{Interface Requirements}

This category covers all requirements related to the service's user interfaces and the monitoring software used for tracking:

- User interface: The service should have a user-friendly interface. Devices used for location detection should not impede the normal activities of the tracked patients. For instance, in case the solution uses tags, tags should be simple, intuitive, lightweight, easy to wear, and acceptable by patients. Moreover, no wire cables should be used.

- Software interface: The location monitoring software must be able to display locations and identifications of the tracked persons in a visualized view, easy to understand and interpret (e.g. house map).

- Location Data granularity: The location software must be able to display general-to-specific location information, users should be able to identify, group, categorize, and customize patients' details as desired.

\section{E. Performance requirements}

This category includes all requirements related to the performance of the service:

- Responsiveness: The service should provide location data in real time basis avoiding delays in critical circumstances.

- Resolution: The estimated location measurements of the service is expected to give at least room-level location resolution for indoor activities and relative location information (e.g. nearby the garden) for outdoors.

- Accuracy: The accuracy requirement is typically related to the quality of the location information. It refers to the quantifiable error distance between the estimated location and the actual location. The service should be able to give high percentage of correct location estimates.

- Interference Avoidance: The service's devices should not be vulnerable to interference from other devices 
worn by the patient or other house's devices (e.g. electric and electronic devices).

- Automatic Fault Detection: The ability to avoid false location detection is required as the service will be used for healthcare applications. The service should be able to automatically detect anomalies in the location data.

- Update Interval: The update interval of the location data should be adjustable to short intervals (e.g. seconds), so that reducing the presence of uncertainty in location sample data.

- Reliability: The location service should maintain its routine functions even in abnormal situations (e.g. power outage); the location service should be reliable, in order to guarantee there is no loss of information.

- Availability: The service should be active and available 24 hours per day; the service should be operate inside/outside buildings no matter the structure state and environmental conditions.

- Scalability: The service should be able to scale well in terms of the number of located patients or the number of tags that can be tracked simultaneously in case the system is based on tags.

\section{F. Product Requirements}

This category includes all requirements related to the deliverable version of the service:

- Cost: Cost-effective service is an important factor for the success and the widely adoption of the service. The cost includes installation, deployment, infrastructure, communication, and maintenance costs.

- Maintainability: The maintenance tasks such as upgrading software, maintaining hardware or expanding the application should not need much more cost and time. When configuring the system, the performed update should not interrupt other parts of the systems. The configuration of the system (software/hardware) should be easy, simple and quick.

- Deployment: The impact on the existing infrastructure (avoiding interference with the existing technologies); environmental considerations (considering the physical layout of the area, amount of metals, etc.); co-existence with existing technologies all these issues should be considered when deploying the service.

- Installation: Installation efforts (cabling installation, sensors installation, power needs, and sensor calibration) should be minimized. The interior design of the deployment environments should not be significantly affected by the installation processes.

- Service Performance: The service should provide means for tracking and measuring its performance, e.g. all notification alarms must be delivered in reasonable time; determined by the level of emergency and according to specific normative documents.

- Integration: The service should have the ability to easily exchange information and integrate with other systems.
- Standardization: The service should be based on industry standards.

- Auto-Functioning: The service should have the ability to be self-initializing, self-calibrating, and selfdiagnostic to ensure reliability. The service should be automatically activated or deactivated.

- Power efficiency: The service should be able to function even with scarce power resources. Moreover, in case the service uses batteries (e.g. tags or wearable sensors), battery replacements should not be very frequent so that batteries should last for as long as possible.

- Usability: The service should just work requiring minimal-to-no training for operation.

- Eco-friendliness: Levels of hazardous or non-hazardous waste caused by the service (for example, pollution caused by the batteries of the tags) should be minimized

- Security: The service should provide information security during data transfer to prevent unauthorized use of location information.

\section{METRICS}

In table I we provide the identified metrics. The metrics are based on evaluation benchmarking provided by the EvAAL initiative [13] and also based on some prior work [14]. In the table the metrics are presented with two evaluation values for each single metric: Target and Threshold. The target is used to depict the desired and expected value whereas the threshold is used to represent the minimum acceptable value for the corresponding metric. For instance, the target value corresponding to the Response time metric is "Within seconds", whereas the threshold is "Less than 5 minutes" which represent the acceptable value for the Response time metric.

As shown in table I the listed metrics are presented to give a formal evaluation method which can be used to quantify the performance of the potential location and tracking services for AAL. It worth mentioning here, we are not looking for metrics for evaluating the performance of remote monitoring systems, instead we are more concerned only about location and tracking metrics for AAL, and therefore, metrics related to, for instance, heart beat sensors or other body attached sensors are not quite relevant to the context of the paper. Furthermore, in the table the "Basic" threshold value, correspond to the use of behavioural models for abnormality detection, refers to the basic functionalities that we expect to have from the use of behavioural models. The basic functionalities include but are not limited to the movement detection (presence), absence of motion, and leaving/entering house. While the "advanced" target value refers to a more advanced functionalities such as abnormal motion sequence or unusual transitions. Moreover, in the table some of the metrics are more important than others; some of the metrics assess mandatory aspects, such as Presence/Absence detection or User Acceptance, while other metrics can be used just to compare the relative merit of different technologies/systems, such as Cost or the use of Wearable Tags. A real world system might have to trade-off 
some of the metrics. Nevertheless, by setting an evaluation/assessment framework, through the metrics, we are defining a set of guidelines that can be used for both technological development and system design.

TABLE I. METRICS

\begin{tabular}{|c|c|c|}
\hline Metric & Target & Threshold \\
\hline Response time & Within seconds & Less than 5 minutes \\
\hline Resolution & Room-level & Room-level \\
\hline Accuracy & $99 \%$ & $90 \%$ \\
\hline Coverage Scope & $\begin{array}{c}\text { Inside house and areas } \\
\text { nearby }\end{array}$ & Inside house \\
\hline Service Output format & $\begin{array}{l}\text { Symbolic (Bedroom, } \\
\text { Kitchen) }\end{array}$ & $\begin{array}{l}\text { Symbolic (Bedroom, } \\
\text { Kitchen) }\end{array}$ \\
\hline Person Identifier & Required & Required \\
\hline $\begin{array}{l}\text { Presence/Absence } \\
\text { indicator }\end{array}$ & at room-level & at house-level \\
\hline $\begin{array}{l}\text { Location Update } \\
\text { Interval }\end{array}$ & Adjustable & Every 5 minutes \\
\hline $\begin{array}{l}\text { Location Sampling } \\
\text { rate/sec }\end{array}$ & $\begin{array}{c}1 \text { sample every } 5 \\
\text { second }\end{array}$ & $\begin{array}{l}1 \text { sample every } \\
\text { minute }\end{array}$ \\
\hline Service Calibration & Self-Calibration & $\begin{array}{l}\text { Easy manual } \\
\text { calibration }\end{array}$ \\
\hline $\begin{array}{l}\text { Service Remote } \\
\text { Communication }\end{array}$ & $\begin{array}{l}\text { Fast connection to } \\
\text { support remote } \\
\text { instructions }\end{array}$ & $\begin{array}{l}\text { Fast connection to } \\
\text { support remote } \\
\text { instructions }\end{array}$ \\
\hline $\begin{array}{l}\text { Remote } \\
\text { Communication Cost }\end{array}$ & Less than Internet Cost & Same as Internet Cost \\
\hline $\begin{array}{l}\text { Resilience to Power } \\
\text { Outage }\end{array}$ & Up to one week & Up to one day \\
\hline Interference avoidance & No Interference & No Interference \\
\hline $\begin{array}{l}\text { Automatic Fault } \\
\text { Detection }\end{array}$ & $\begin{array}{c}\text { Required, with } \\
\text { notification to remote } \\
\text { assistance service }\end{array}$ & $\begin{array}{l}\text { Required, with local } \\
\text { indication }\end{array}$ \\
\hline Battery life time & more than 1 year & 6 months \\
\hline \# Location devices & One per house & Two per house \\
\hline Use of Wearable tags & No tags & $\begin{array}{c}\text { Easy to carry and } \\
\text { wear }\end{array}$ \\
\hline $\begin{array}{l}\text { Installation } \\
\text { Complexity }\end{array}$ & Low & Low \\
\hline $\begin{array}{l}\text { Remote/Local } \\
\text { Computation }\end{array}$ & Local & Remote \\
\hline $\begin{array}{l}\text { Use of behavioural } \\
\text { models for } \\
\text { abnormality detection }\end{array}$ & Advanced & Basic \\
\hline $\begin{array}{l}\text { User's Movement } \\
\text { History }\end{array}$ & $\begin{array}{l}\text { Up to } 1 \text { year for } \\
\text { behavioural models }\end{array}$ & Up to 6 month \\
\hline \# Tracked Persons & Multiple & One per house \\
\hline User acceptance & Required & Required \\
\hline Service Cost & Cheap & Cheap \\
\hline
\end{tabular}

\section{A. WiFi Fingerprinting}

Due to the wide availability of WiFi networks, location services based on $\mathrm{WiFi}$ fingerprinting have gained more attention in the last few years [15]. WiFi fingerprinting is one of the most mature techniques, with several commercial systems available, and that systems based on this techniques can effectively benefit from existing infrastructures as $\mathrm{WiFi}$ networks are becoming ubiquitous. The technique estimates the locations based on the received signal strength (RSS) collected from WiFi access points. Thus, it does not demand additional hardware than what already exists at houses. However, issues like collecting, measuring, and calibrating the location fingerprints are still great challenges facing this technique. Ekahau [8] and Microsoft RADAR [9] are some of the location systems based on WiFi Fingerprinting.

\section{B. Ultrasound-based}

Location services based on ultrasound consist of a set of ceiling-mounted receivers that detect ultrasound signals from tags, at user-defined time interval, to calculate distances using time-of-flight. This technique has the potential to provide good accuracy and that the fundamental devices are cheap. However, the synchronization of the location sensors and the high installation complexity are major challenges facing this technique. Location systems that make use of ultrasound include Active bat [10], Crickets [16], Sonitor [17], and Dolphin [18].

Table II shows the evaluation of the aforementioned location measurement techniques against the proposed metrics. In the table "+" depicts that the technique has satisfied the corresponding metric while "_“ depicts unsatisfied. For each technique, there is a pair of values, represented as (target, threshold) respectively, to compare the technique against the identified values of the corresponding metric.

As illustrated in Table II, the two location techniques used in the comparison study are not designed to fully comply with all the requirements of AAL. For instance, the Coverage scope requirement is not fully supported by both techniques. In nondense urban environments, unless additional location sensors/devices have been deployed (e.g. additional WiFi access points and ultrasound receivers) the Coverage Scope requirement will not be fully satisfied. Moreover, WiFi fingerprinting requires additional effort for calibrating the neighbourhoods' areas, in order to provide the required coverage, which means an extra deployment complexity. The 
ideal location service for AAL should be able to provide the service with less effort and minimum number of location sensors/devices. In addition, the resilience for power outage is not considered by both techniques. Power outage is mostly occurring in houses and residential environments especially in rural areas. The two techniques do not provide a reasonable solution for this requirement. Furthermore, the installation complexity for the two techniques is too high. Location service for AAL has to be easy to install in terms of time, cost, and effort. Also the requirement for automatic fault detection is not fully supported in both techniques. Handling and avoiding anomalies and outliers in the location data is still an issue in both techniques. Additionally, the overall cost for the two techniques is relatively high, reducing the likelihood of using AAL services by large sector of people. Moreover, neither of the two techniques makes use of behavioural models for abnormality detection. The aggregated location data can be utilized to build realistic models that represent the typical behaviours of the assisted persons and then use these models for detecting unusual behaviours and thus voiding critical situations in advance.

TABLE II. WIFI FINGERPRINTING VS ULTRASOUND-BASED COMPARISON

\begin{tabular}{|l|c|c|}
\hline \multicolumn{1}{|c|}{ Metric } & $\begin{array}{c}\text { WiFi } \\
\text { Fingerprinting }\end{array}$ & Ultrasound-based \\
\hline Response time & $(+,+)$ & $(+,+)$ \\
\hline Resolution & $(+,+)$ & $(+,+)$ \\
\hline Accuracy & $(+,+)$ & $(+,+)$ \\
\hline Coverage Scope & $(-,+)$ & $(-,+)$ \\
\hline Service Output Format & $(+,+)$ & $(+,+)$ \\
\hline Person Identifier & $(+,+)$ & $(+,+)$ \\
\hline Presence/Absence indicator & $(+,+)$ & $(+,+)$ \\
\hline Location Update Interval & $(+,+)$ & $(+,+)$ \\
\hline Location Sampling rate/sec & $(+,+)$ & $(+,+)$ \\
\hline Service Calibration & $(-,-)$ & $(+,+)$ \\
\hline $\begin{array}{l}\text { Service Remote } \\
\text { Communication }\end{array}$ & $(+,+)$ & $(+,+)$ \\
\hline Remote Communication Cost & $(-,+)$ & $(-,+)$ \\
\hline Resilience to Power Outage & $(-,-)$ & $(-,-)$ \\
\hline Interference avoidance & $(-,-)$ & $(+,+)$ \\
\hline Automatic Fault Detection & $(-,-)$ & $(-,-)$ \\
\hline Battery life time & $(-,-)$ & $(-,-)$ \\
\hline \# Location Devices & $(-,-)$ & $(-,-)$ \\
\hline Use of Wearable tags & $(-,+)$ & $(-,+)$ \\
\hline Installation Complexity & $(-,-)$ & $(-,-)$ \\
\hline Remote/Local Computation & $(+,+)$ & $(+,+)$ \\
\hline User's Movement History & $(+,+)$ & $(+,+)$ \\
\hline \# Tracked Persons & $(+,+)$ & $(+,+)$ \\
\hline User acceptance & $(-,-)$ & $(-,-)$ \\
\hline $\begin{array}{l}\text { Use of behavioural models } \\
\text { for abnormality }\end{array}$ & $(-,-)$ & $(-,-)$ \\
\hline Service Cost & $(-,-)$ & \\
\hline
\end{tabular}

\section{CONCLUSION}

In this paper, we discussed the requirements for $\mathrm{AAL}$ location and tracking services. We presented a set of metrics to be used in evaluating the potential location services for AAL. The identified requirements and metrics are based on a typical scenario for AAL system. Some of the selected metrics are based on evaluation benchmarking provided by the EvAAL initiative [13]. Two of the existing location techniques have been evaluated through the use of the proposed metrics. The results showed that most of the requirements are not fully considered by these techniques and, therefore they are not quite adequate for AAL. Further research is needed to bridge the gap between the current solutions and the real location and tracking requirements for $\mathrm{AAL}$.

One possible research direction is the use of behavioural models that represent the typical activity behaviour of the assisted persons. These models could be used significantly to provide real time information about the deviation from the usual behaviour for each person and then avoiding emergency situations in advance. Many reasoning and inferring techniques can be used to support the applicability of using location patterns in behavioural modeling. However, issues like how to infer behaviours from location raw data, how to form activity logic specifications (i.e. context attributes of an activity), and how to deal with noisy and insufficient location datasets are considered great challenges facing the inclusion of such facility.

In this paper, we are contributing to the definition of a generic evaluation framework. The framework is to be used as reference for potential AAL location services. The framework is not only for evaluation purposes but, also for providing guidelines for technological developments and system design. Additional evaluation and comparison studies are required to completely define all the requirements.

\section{ACKNOWLEDGMENT}

The authors would like to thank the reviewers for their valuable comments and suggestions.

This work was supported by the FEDER program through the COMPETE and the Portuguese Science and Technology Foundation (FCT), within the context of the AAL4ALL (COMPETE 13852) and FCOMP-01-FEDER-0124-022674 projects.

\section{REFERENCES}

[1] K. Giannakouris, "Ageing characterises the demographic perspectives of the European societies," Eurostat, Statistics in focus, vol. 72, 2008.

[2] E. Strickland. (2012, FEBRUARY ) Web Guru for the Blind. IEEE SPECTRUM. 51-53. Available: www.SPECTRUM.IEEE.ORG

[3] World Health Organization. (2012, World Health Day 2012 - Ageing and health Demographic changes are accompanied by new challenges. Available: http://www.who.int/en/

[4] H. Sun, De Florio, V,Gui, N, Blondia, C., "Promises and challenges of ambient assisted living systems," in ITNG '09 Proceedings of the 2009 Sixth International Conference on Information Technology: New Generations, Washington, DC, USA, 2009, pp. 1201-1207.

[5] H. Liu, Darabi, H,Banerjee, P,Liu, J., "Survey of wireless indoor positioning techniques and systems," IEEE Transactions on Systems, 
Man, and Cybernetics, Part C: Applications and Reviews, , vol. 37, pp. 1067-1080, 2007.

[6] Y. Gu, Lo, A,Niemegeers, I., "A survey of indoor positioning systems for wireless personal networks," IEEE Communications Surveys and Tutorials, vol. 11, pp. 13-32, 2009

[7] G. Borriello and J. Hightower, "A survey and taxonomy of location systems for ubiquitous computing," IEEE Computer, vol. 34, pp. 57-66, 2001.

[8] Ekahau Company. (17/2/2012). Real-Time Location System (RTLS). Available: http://www.ekahau.com/

[9] P. Bahl and V. N. Padmanabhan, "RADAR: An in-building RF-based user location and tracking system," in Proc. IEEE INFOCOM 2000. Nineteenth Annual Joint Conference of the IEEE Computer and Communications Societies. , Tel Aviv, Israel, 2000, pp. 775-784.

[10] J. Hightower and G. Borriello, "Location systems for ubiquitous computing," IEEE Computer, vol. 34, pp. 57-66, 2001.

[11] R. Want, Hopper, A,Falcao, V,Gibbons, J., "The active badge location system," ACM Transactions on Information Systems (TOIS), vol. 10, pp. 91-102, 1992.

[12] B. P. L. Lo, Wang, J.L,Yang, G.Z., "From imaging networks to behavior profiling: Ubiquitous sensing for managed homecare of the elderly," in Proc. 3rd Int'l Conf. on Pervasive Computing, Munich, Germany, 2005.
[13] EvAAL. (2012, Evaluating AAL Systems through Competitive Benchmarking. Available: http://evaal.aaloa.org/currentcompetition/annex2012-track-localization

[14] E. Chan, Baciu, G,Mak, SC, "Using Wi-Fi signal strength to localize in wireless sensor networks," in IEEE CMC: International Conference on Communications and Mobile Computing, Kunming, Yunnan, China, 2009, pp. 538-542.

[15] M. Rodrigues, Vieira, LFM,Campos, MFM, "Fingerprinting-based radio localization in indoor environments using multiple wireless technologies," in 22nd International Symposium on Personal, Indoor and Mobile Radio Communications (PIMRC), Brazil, 2011, pp. 12031207.

[16] N. B. Priyantha, Chakraborty, A,Balakrishnan, H., "The cricket locationsupport system," in Proc. of the Sixth Annual ACM International Conference on Mobile Computing and Networking (MOBICOM), Boston, Massachusetts, USA, August 2000, pp. 32-43.

[17] Sonitor Technologies. (17/2/2012). Available: http://www.sonitor.com/

[18] Y. Fukuju, Minami, M,Morikawa, H,Aoyama, T., "DOLPHIN: An autonomous indoor positioning system in ubiquitous computing environment," in Proc. of the IEEE Workshop on Software for Future Embedded Systems (WSTFES'03), Hakodate, Japan, 2003, pp. 53-56. 